\title{
Optimized Image Compression through Artificial Neural Networks and Wavelet Theory
}

\author{
Raghvendra Pratap Singh \\ MMMEC, Gorakhpur \\ UP, India
}

\author{
Choudhary Mahfooz Alam \\ JMI, New Delhi \\ India
}

\author{
J P Saini \\ MMMEC \\ Gorakhpur, UP \\ India
}

\begin{abstract}
Many techniques have been developed for image compression. An efficient image compression technique promises to give high compression ratio, maintaining the quality of the image. The paper proposes an image compression technique which combines both Artificial Neural Networks and Wavelet theory to optimize the compression ratio and peak signal to noise ratio. Results show that high compression ratio is achievable as per requirement, maintaining good reconstruction quality.
\end{abstract}

\section{General Terms}

Image Compression

\section{Keywords}

ROI, SPIHT, FFN.

\section{INTRODUCTION}

Storing and transmitting high resolution images over a transmission channel with limited bandwidth are very challenging. So there is a consistent need of research work to be done in the area of image compression. Image compression addresses the problem of reducing the amount of data required to represent a digital image. The underlying basis of the reduction process is the removal of redundant data. From a mathematical view point, this amounts to transforming a 2-D pixel array into a statistically uncorrelated data set. The transformation is applied prior to storage or transmission of the image. At some later time, the compressed image is decompressed to reconstruct the original image or an approximation of it. In practice, there is two types of compression; namely lossless and lossy compression. In lossless compression, the reconstructed image is exactly same as the original. The criterion of performance is not perception by the human eye but two physical quantities; namely the mean square error and the peak signal to noise ratio. They are calculated as follows:

$$
M S E=\frac{1}{M \times N} \sum_{i=0}^{M-1} \sum_{j=0}^{N-1}[F(i, j)-f(i, j)]^{2}
$$

where $\mathrm{F}$ represents original image of size $\mathrm{M} \times \mathrm{N}$ and $\mathrm{f}$ is the reconstructed image of same size.

$P S N R=10 \log _{10} \frac{1}{N M S E}$

Where NMSE is the normalized mean square error and PSNR is measured in decibels.

For lossless compression, the MSE is zero and the PSNR is infinity. However the majority of applications in image processing do not require that the reconstructed image and the original should be exactly alike. This comes as an advantage for most applications, because we can compress the original image to a value that would be suitable for the desired requirement. Hence comes lossy compression into the picture. The advantage of lossy compression is to save a substantial amount of memory while storing or transmitting images over a communication channel. The effect of compression comes as image distortion in the reconstructed image. In lossy compression, the image quality definitely degrades due to loss of information. Nevertheless, lossy compression techniques generally offer much higher compression ratios than the lossless technique [1], [10].

In this paper a technique for image compression is proposed which incorporates the advantages of both lossless and lossy techniques.

\section{ARTIFICIAL NEURAL NETWORK FOR IMAGE COMPRESSION}

The image is divided into blocks of $\mathrm{m}$ by 1 pixels before performing a suitable image transform to the pixels on the block. Through this step, complexity of the computation and the required memory space to store the calculation temporarily can be reduced thus resulting in faster transformation time [2]. Each of the coefficients in the transformed blocks with the corresponding horizontal position, $\mathrm{x}$ and vertical position, $\mathrm{y}$ will then be propagated through the ANN block by block sequentially .The inputs to the ANN will be the spatial $\mathrm{x}$ and $\mathrm{y}$ coordinates while the output for the network will be relevant transform coefficients[3].

At the decompression stage, the values of the coefficients in each block can be recomputed by setting the input of the neural network to the specific spatial coordinates under consideration. Subsequently, the network will produce the equivalent transformed coefficients which are then inversed transformed to get back original pixel value. The effectiveness of the compression algorithm is measured using two parameters which are the PSNR given by (2) for assessing the quality of the decompressed image and the image compression ratio given by (3).

Compression Ratio $=\frac{\text { Original Image File Size }}{\text { Compressed Image File Size }}$

Multilayer Perceptron (MLP) is used for image compression with neural networks [4]. In this method, an MLP network is developed. In this network there are three layers named input layer, hidden layer and output layer. The input is given to the input layer, this is the frequency value of image that means the pixel vale of original image is applied to input neurons. The number of neurons at the input is same as the number of pixels in the image. The number of neurons at the middle layer (hidden layer) is lesser than number of neurons at the input layer, to achieve compression. The compression rate can be increase by adding more number of hidden layers [5] Number of neurons at the output layer is similar to the number 
of neurons at the input layer to approximate original image. Therefore the compression is achieved at the hidden layer and image reconstruction is done at the output layer. Back propagation training algorithm has been used to correct the weights of this network. The fundamental for image compression using all type of neural networks is to map the set of inputs to a set of outputs through a hidden layer; the number of neurons at hidden layer should always be smaller than input and output neurons [6]. In this process, it is necessary to have output as similar as possible to the inputs. Thus the hidden layer can represent the input layer effectively when the size of hidden layer is smaller than that of input layer. Then the hidden layer gets data from the input layer.

\section{PROPOSED IMAGE COMPRESSION TECHNIQUE}

The MLP neural network approach for the purpose of image compression has led to the problem of block effect in the reconstructed image. Especially by increasing compression rate the problem with reconstructed image arises in the sense that quality is degraded [7]. It provides the idea to make use of the interesting results of wavelet transformation in the imagery field. For this purpose, wavelet coefficients acquired by the image decomposition are taken as the inputs of the neural networks instead of the gray levels of the image [8].

The same approach described in previous section is used, but the difference is that here first apply the wavelet transform decomposition on the original image. Then follow the training step. From the input layer to output layer a feed-forward propagation learning algorithm have been applied. Backforward propagation learning is used to update the weights and parameters. In this back-propagation learning algorithm the weights are moved in the negative gradient direction. This direction of the negative gradient is the direction of the most rapid performance decrease. Despite this offline gradient descent training algorithm can make the computation very complex and heavy, still use it to compare the advantage and disadvantage between traditional neural network and MLP network with wavelet coefficients [9]. So gradients descent algorithm is used to train the networks.

In order to compress the image, first, it's required to segment it in a set of $\mathrm{m}$ blocks 1 by 1 pixels. These blocks are used as inputs for the designed neural network. A three layer feedforward neural network is used: an input layer with $\mathrm{m}$ neurons with $1 \times 1$ block pixels, an output layer with $m$ neurons and a hidden layer with a number of neurons smaller than $\mathrm{m}$. The network is trained in order to reproduce in output the information given in input. The input block is denoted by $\mathrm{X}=(\mathrm{x} 1, \ldots ., \mathrm{xm})$ and the output of the network by $\mathrm{Y}=(\mathrm{y} 1, \ldots \ldots$, $\mathrm{ym})$. At the end of the training the requirement is to get $\mathrm{Y}=\mathrm{X}$ for every block presented to the network.

\section{IMPLEMENTATION OF THE PROPOSED TECHNIQUE}

The approach of image compression enhancement is based on the neural network with wavelet coefficients. Back propagation learning algorithm has been used for the training process. The image compression by MLP neural networks (ANN) starts with image decomposition, in this process segmentation of image in stationary blocks is done. The size of the block could be chosen by the user. The effect of this operation is shown in fig. 1 .

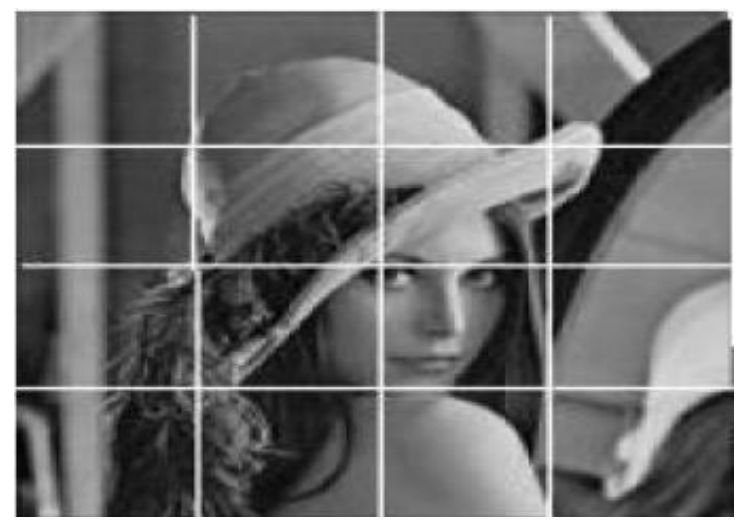

Fig. 1 Effect of Image Segmentation

The training of the network is self adjustable while applying back-propagation. The training data contain the vector representation for one block of the image. The initialization of the network parameters started randomly. Thereafter training has started. Training required a set of prototypes and also target for the learning of the network behaviour. The parameters of the network are iteratively adjusted during the training stage. The training task has been repeated until the verification criterion is satisfied. The number of iteration is set by user. Whenever the image is compressed by MLP with wavelet coefficients the similar approach of the neural networks are used but first of all wavelet transform decomposition is applied on the original image. Then follow training step and finally apply inverse wavelet transformation to obtain the reconstructed compression image.

\section{RESULTS}

The results of the compression technique applied to the Malaviya ji image (fig. 2) is shown in fig.3. The three images have taken under consideration. Table 1 shows the results of compression for the images of Malaviya ji, Lena and Cameraman by MLP with wavelet coefficients.

The Comparison of PSNR for Compression by only ANN and Compression by MLP with Wavelet for Malaviya ji image is shown in fig. 4. 


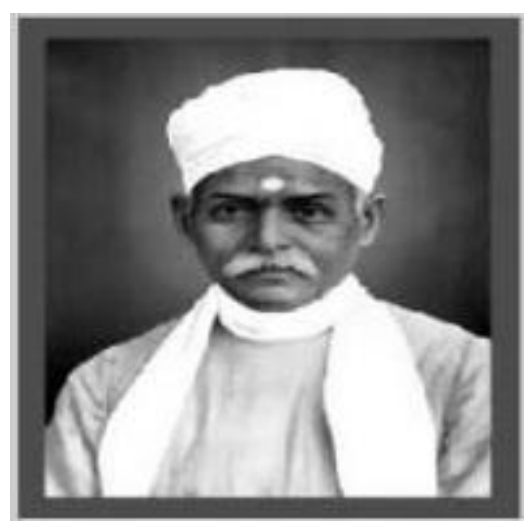

Fig. 2 Malaviya ji Image

Table 1: Compression ratio and PSNR

\begin{tabular}{|c|c|c|c|c|}
\hline Image & Compresssion Ratio & $\begin{array}{l}\text { No. of Hidden } \\
\text { Neurons }\end{array}$ & PSNR (dB) & MSE \\
\hline \multirow[t]{4}{*}{ Malaviya ji } & $25 \%$ & 16 & 21.0143 & 153.4669 \\
\hline & $50 \%$ & 32 & 21.0119 & 154.8085 \\
\hline & $75 \%$ & 48 & 20.9375 & 160.9141 \\
\hline & $87.5 \%$ & 56 & 20.0478 & 181.9356 \\
\hline \multirow[t]{4}{*}{ Lena } & $25 \%$ & 16 & 19.3312 & 120.2882 \\
\hline & $50 \%$ & 32 & 19.2483 & 110.0423 \\
\hline & $75 \%$ & 48 & 19.1624 & 107.7509 \\
\hline & $87.5 \%$ & 56 & 19.3894 & 106.2011 \\
\hline \multirow[t]{4}{*}{ Cameraman } & $25 \%$ & 16 & 18.7191 & 283.4723 \\
\hline & $50 \%$ & 32 & 18.3249 & 255.2437 \\
\hline & $75 \%$ & 48 & 18.6384 & 249.6384 \\
\hline & $87.5 \%$ & 56 & 18.5645 & 246.8092 \\
\hline
\end{tabular}


Compressed Image
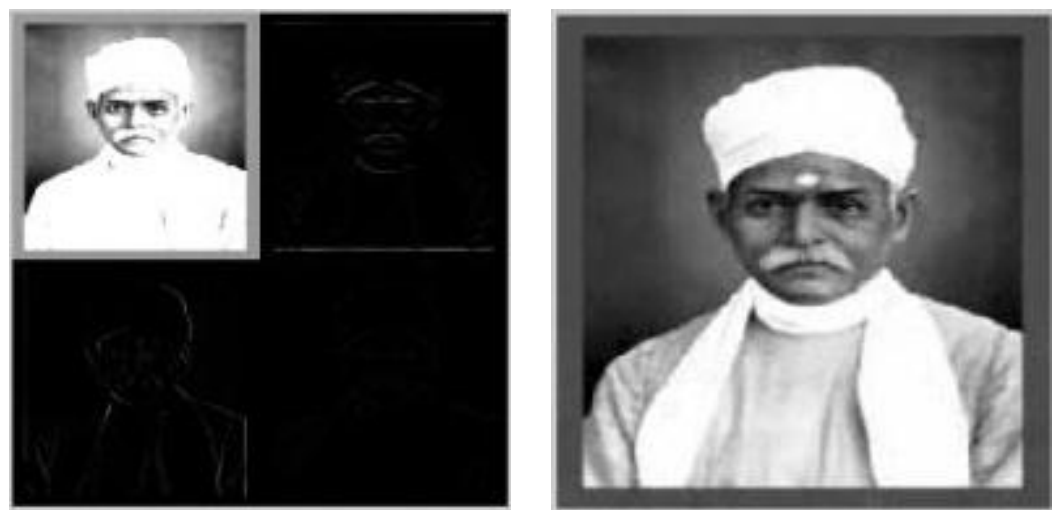

(a)
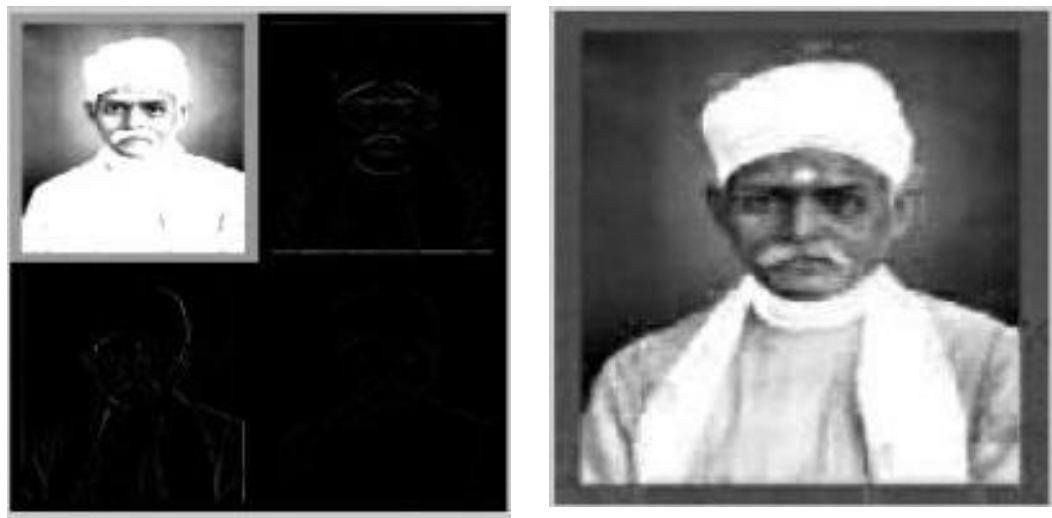

(b)

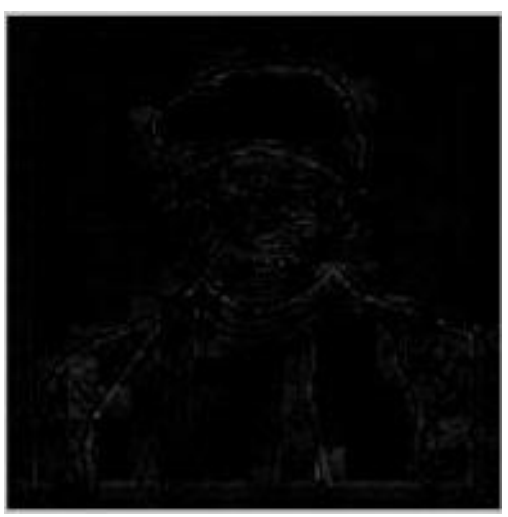

Error Image

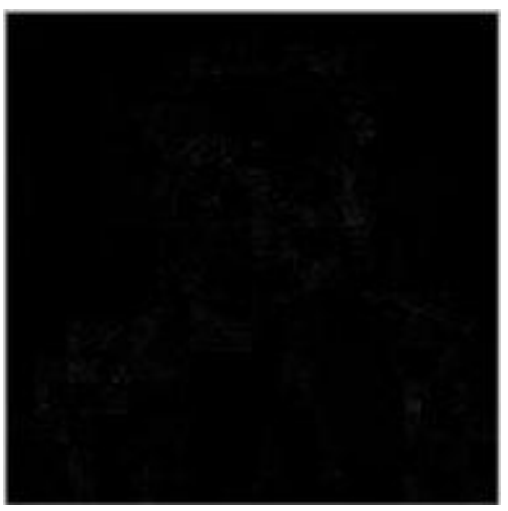

\section{(a)}

\section{)}

Fig. 3(a) Results for Compression Ratio 75\% (b) Results for Compression Ratio 87.5\%

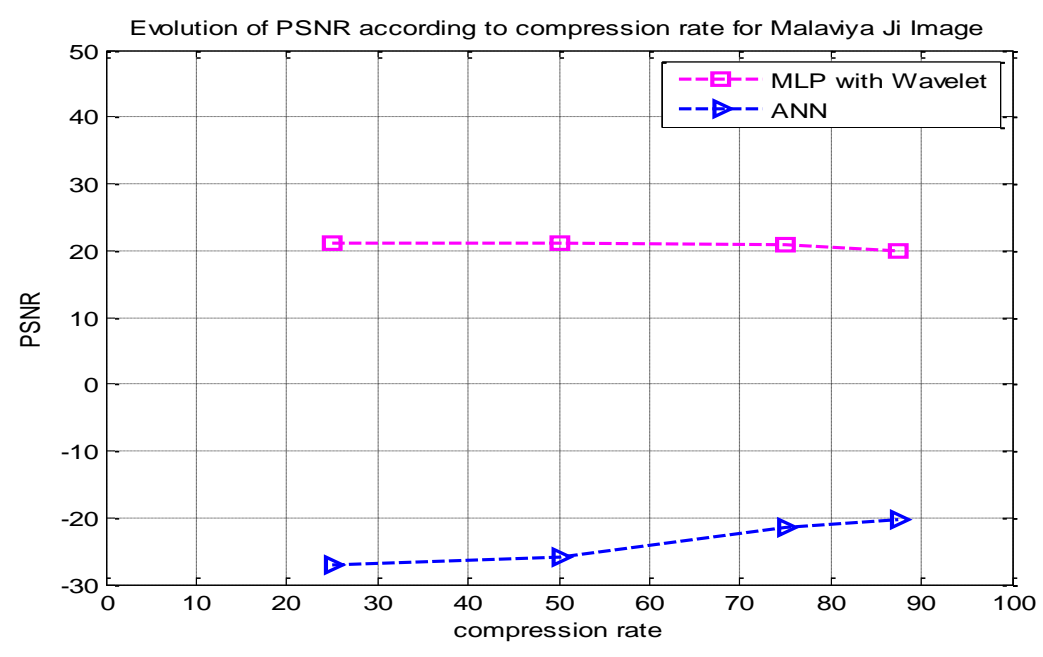

Fig. 4 Comparison of PSNR for Compression by only ANN and by MLP with Wavelet 


\section{CONCLUSION}

The compression results vary for the different images because number of bits that are required to represent an image differs for all three images, but all images shows better compression performance when wavelets are used with MLP as compare to classical MLP approach. The algorithm gives good results for the compression rate up to $87.5 \%$ but beyond this limit it gives undesirable results. The algorithm has flexibility in the sense that compression ratio can be improved as per the requirement by increasing the number of hidden neurons. The reconstruction quality can be improved by increasing the iterations. Another positive feedback have been obtained that the PSNR value is good and have very less variation by increasing the compression rates from $25 \%$ to $87.5 \%$. According to the results the PSNRs are high enough, even by increasing the compression rate. So, if higher compression ratio is needed with good reconstruction quality (more than $75 \%$ compression ratio) the use of wavelet decomposition with MLP (i.e MLP with wavelet coefficients) provides better result. It comprises the advantages of both ANN and Wavelet for the compression of digital images.

\section{REFERENCES}

[1] R. C. Gonzalez and R. E. Woods, "Digital Image Processing", Addision Wesley, New York, USA, 1981.

[2] S. Haykin, "Neural Networks- A Comprehensive Foundation", 2nd Edition, Pearson Publication, 2004.

[3] Vikash Kumar, Jitu Sharma and Shahanaz Ayub. Article: Image Compression using FFN for ROI and SPIHT for background. International Journal of Computer Applications 46(18):30-35, May 2012.

[4] D. Kornreich, Y. Benbenisti, H.B. Mitchell, P. Schaefer, "A High Performance Single Structure Image Compression Neural Network", IEEE Trans. Aerospace Electronic Systems, p.p. 1060-1073, 1997.

[5] A. Namphol, S. Chin, M. Arozullah, "Image Compression with A Hierarchical Neural Network", IEEE Trans. Aerospace Electronic Systems, p.p. 326337, 1996.

[6] Dong Changhong, "Neural Networks and Applications", $2^{\text {nd }}$ edition, Beijing, China: National Defence Industry, p.p. 14-120, 2009.

[7] C. Ben Amar and O. Jemai, "Wavelet Networks Approach for Image Compression", GVIP Special Issue on Image Compression, ICGST, p.p. 15-23, 2007.

[8] Weiwei Xiao, Haiyan Liu, "Using Wavelet Networks in Image Compression", Seventh International Conference on Natural Computations, p.p. 700-704, 2011.

[9] Hamdy S, Soliman, Mohammed Omari, "A Neural Network Approach to Image Compression Applied Soft Computing”, Vol. 6, Issue 3, p.p.258-271, 2006.

[10] Rufai, Awwal Mohammed, Gholamreza Anbarjafari, and Hasan Demirel. "Lossy image compression using singular value decomposition and wavelet difference reduction.” Digital Signal Processing (2013). 